\title{
Osteosarcoma outcomes at a South African tertiary hospital
}

\author{
L Lisenda, ${ }_{1}^{1} \mathrm{MB}$ ChB BAO, MMed, MRCS, FC Orth (SA); Z A Linda, ${ }^{1} \mathrm{MB} B C h$, FC Orth (SA); F P J Snyman, ${ }^{2}$ MB BCh, MMed; \\ R D Kyte, ${ }^{1,2,3}$ MB BCh, FC Orth (SA), M Lukhele, ${ }^{1}$ MB BCh, MMed, FC Orth (SA) \\ ${ }^{1}$ Division of Orthopaedic Surgery, Charlotte Maxeke Johannesburg Academic Hospital and Faculty of Health Sciences, University of the \\ Witwatersrand, Johannesburg, South Africa \\ ${ }^{2}$ Milpark Hospital, Johannesburg, South Africa \\ ${ }^{3}$ Wits Donald Gordon Medical Centre, Johannesburg, South Africa
}

Corresponding author: L Lisenda (lisendal@hotmail.com)

Background. Osteosarcoma is the most common primary malignant bone tumour. There is a high incidence of late presentation in the developing world, posing additional challenges in the treatment of this aggressive disease.

Objective. To evaluate clinical outcomes of patients with osteosarcoma at Charlotte Maxeke Johannesburg Academic Hospital (CMJAH), a tertiary hospital in South Africa, and compare these with similar studies in the developing world.

Methods. This was a retrospective study of 61 patients treated at CMJAH between 2007 and 2011, with a minimum follow-up of 1.3 years (range $1.3-6.3$ ).

Results. An average of 4.5 months elapsed before patients were first seen at the CMJAH tumour unit. Fifty-eight patients (95.1\%) initially sought conventional medical care. Three patients (4.9\%) presented with pathological fractures. All the patients underwent biopsy, performed an average of 3 weeks after arrival at the tumour unit. In most cases the delay was due to limited access to magnetic resonance imaging. Most patients ( $n=41,67.2 \%)$ were at Enneking stage 2B, $4(6.6 \%)$ were at stage $2 \mathrm{~A}$ and $16(26.2 \%)$ were at stage 3 . Of the patients, $13(21.3 \%)$ underwent limb salvage procedures, $33(54.1 \%)$ had amputations, $4(6.6 \%)$ refused further treatment and $11(18.0 \%)$ received palliative care only; 55 patients $(90.2 \%)$ received chemotherapy. Two patients developed local recurrence, one of whom had an amputation and the other further wide excision. Two patients received palliative radiotherapy. Of the patients, $82.0 \%$ were HIV-negative, $4.9 \%$ HIVpositive and the rest of unknown status. At the time of the study, all but two patients, who came from other countries, were traceable or known to have died. Our overall 1-year and 5-year survival rates were $62.7 \%$ (95\% confidence interval (CI) $49.1-73.9)$ and $38.1 \%(95 \%$ CI 24.6 - 51.4), respectively. Male patients and those with a higher Enneking stage had a poorer prognosis.

Conclusion. Although most patients sought conventional medical care, unacceptable delays worsened survival. However, our survival rates are better than those in other developing countries. We advocate that professional, public and political awareness of osteosarcoma be improved as a matter of urgency, to facilitate rapid tertiary referral and expedite management.

S Afr Med J 2017;107(9):754-757. DOI:10.7196/SAMJ.2017.v107i9.11424

Osteosarcoma is the most common primary malignancy of bone. ${ }^{[1]}$ It is relatively rare, however, with an incidence of 4 per million population per annum. ${ }^{[1,2]}$ The peak incidence is in the second decade of life, with a smaller peak in the 7 th and 8 th decades, these cases usually being secondary. ${ }^{[2-4]}$ The tumour is more common in males than in females $^{[5,6]}$ and has a higher prevalence among blacks than among whites. ${ }^{[7]}$ The cause is unknown, but individuals with germline mutations in the retinoblastoma $(R b)$ and tumour protein p53 (TP53) genes have been shown to be at increased risk. ${ }^{[8,9]}$

Survival was as low as $5 \%$ in the 1950 s, with a 5 -year survival rate of only $22 \%$, most patients undergoing amputation and adjuvant radiation only or pioneering chemotherapy. ${ }^{[10]}$ During the 1970s there was a marked improvement in 5-year survival rates, which were reportedly as high as $81.6 \%$ in one study, ${ }^{[11,12]}$ largely owing to significant advances in chemotherapy, with utilisation of high-dose and multiagent regimens. In addition, tumour necrosis induced by neoadjuvant chemotherapy assisted in reducing the size of the surgical margin required for wide tumour excision, facilitating successful limb salvage without negatively affecting overall survival. ${ }^{[13,14]}$

Unfortunately, improvements in osteosarcoma management that are routine in the developed world are still not common in developing countries, because of limitations in human resources and lack of equipment and access to new and more effective chemotherapy regimens. Furthermore, it has been reported that cultural beliefs and reliance on traditional medicine in developing countries have resulted in late presentation to conventional hospitals, resulting in poorer outcomes. ${ }^{[5,15-17]}$ Reliable data from clinical outcome and epidemiological studies in developing countries are also scarce. ${ }^{[17]}$ Consequently, accurate benchmarking of evidence-based management protocols is extremely difficult to achieve. Muthupei and Mariba ${ }^{[15]}$ reported a 5 -year survival rate of $7.5 \%$ in their 2000 study of 66 osteosarcoma patients at a tertiary hospital in South Africa (SA), which is comparable to outcomes from the 1950s in developed countries. ${ }^{[10]}$ In 2010, Shipley and Beukes ${ }^{[16]}$ reported on 30 patients with osteosarcoma treated at another SA tertiary institution. Half of their patients presented with metastases. ${ }^{[16]}$ The majority of patients in these studies from developing countries presented with advanced disease, to which the authors attributed their poor outcomes compared with the far better survival rates in developed countries..$^{[5,15-17]}$ Noor et al. ${ }^{[17]}$ had similar findings in a study in Cambodia, with a 5 -year survival rate of only $8 \%$, citing late presentation and cultural preferences as the main factors responsible for the poor outcomes. ${ }^{[17]}$

\section{Objective}

To evaluate clinical outcomes of patients with osteosarcoma at Charlotte Maxeke Johannesburg Academic Hospital (CMJAH), a tertiary hospital in SA, and compare these with similar studies 
from the developing world to evaluate factors contributing to any differences encountered.

\section{Methods}

This was a retrospective study of 61 patients treated for osteosarcoma at the CMJAH tumour unit over the 5-year period January 2007 December 2011. The minimum follow-up was 1.3 years (mean 3.1, range $1.3-6.3$ ). CMJAH is a level 1 trauma centre and one of the two main teaching hospitals of the University of the Witwatersrand, catering not only for the city of Johannesburg but also for Gauteng Province and neighbouring provinces and countries. Only patients with biopsy-confirmed osteosarcoma were included in the study.

Ethics clearance to conduct the study was obtained from the University of the Witwatersrand (ref. no. M150783). The data were obtained from theatre registry and patient records. All patients admitted to the tumour unit had a comprehensive form (available from the first author at lisendal@hotmail.com) completed on admission, throughout the course of admission and during followup. The tumour form had three main sections: (i) history and clinical findings; (ii) investigations (blood tests and radiography) and procedures (biopsy/surgical operations) done; and (iii) follow-up. All patients received a standard osteosarcoma work-up consisting of blood investigations and imaging before biopsy and then definitive treatment. Most patients were followed up at orthopaedic and oncology outpatient clinics, and those who had defaulted were contacted telephonically.

The main outcome measures were demographics, tumour stage according to the Enneking staging system, and treatment options offered (surgery (amputation and limb salvage), chemotherapy, radiotherapy) and survival. Survival was calculated in years from the date of presentation to the time of the study. Kaplan-Meier analysis was used to portray survival functions. Equality of survivor functions was investigated using the log-rank test.

\section{Results}

A total of 61 patients were included in the study (41 males and 20 females, male/female ratio 2:1). The mean age was 19.4 years (range 7 -48). Most of the patients $(n=36,59.0 \%)$ were in their second decade, followed by the third decade $(n=13,21.3 \%)$ and then

Table 1. Histological typing (World Health Organization classification) $(N=61)$

\begin{tabular}{ll}
\hline Type & Patients, $\boldsymbol{n}(\%)$ \\
\hline Conventional & $57(93.4)$ \\
Chondroblastic & 9 \\
Fibroblastic & 5 \\
Osteoblastic & 22 \\
Not specified & 19 \\
Periosteal & $1(1.6)$ \\
Pariosteal & $2(3.3)$ \\
Telangiectatic & $1(1.6)$ \\
Small cell, low-grade surface, secondary & 0
\end{tabular}

the first $(n=5,8.2 \%)$. The fourth and fifth decades accounted for 4 $(6.6 \%)$ and $3(4.9 \%)$ patients, respectively.

Of the patients, $43(70.5 \%)$ and $15(25.6 \%)$ initially sought care from their local clinic and general practitioner, respectively, before being referred to the tumour unit. Only 3 (4.9\%) went to a traditional healer before being referred. On average, it took 4.5 months for patients to be seen in the tumour unit for the first time. Most patients $(n=42,68.9 \%)$ were from the CMJAH catchment area of Gauteng Province, the other SA provinces and other countries accounting for $16(26.2 \%)$ and $3(4.9 \%)$, respectively.

The majority of the patients $(n=58,95.1 \%)$ presented with a painful swollen limb and the rest $(n=3,4.9 \%)$ with pathological fractures. The tibia was the most common site of involvement, accounting for 30 cases (49.2\%), with the femur and humerus accounting for 19 $(31.1 \%)$ and $12(19.7 \%)$, respectively. Only 3 patients (4.9\%) were HIV-positive; 8 (13.1\%) were of unknown status, and the rest $(n=50$, $82.0 \%)$ were HIV-negative.

Biopsy was done at a mean of 3 weeks after presentation. In most cases, the delay was because magnetic resonance imaging (MRI) was not readily available. The majority of the patients $(n=41,67.2 \%)$ were at Enneking stage 2B, $4(6.6 \%)$ were at stage $2 \mathrm{~A}$, and $16(26.2 \%)$ presented with metastases (stage 3). Conventional osteosarcoma was the most common histological type, accounting for $93.4 \%$ of cases (Table 1 ).

In 46 cases $(75.4 \%)$ surgery was undertaken with the aim of achieving wide local resection margins (Table 2). Of these 46 patients, 13 (28.3\%) had limb salvage procedures and 33 (71.7\%) had amputations. Four patients (6.6\%) refused any form of treatment despite multidisciplinary counselling and were discharged from the hospital for home-based care, and the rest $(n=11,18.0 \%)$ received palliative care (palliative care only without amputation) because they presented late (these included two patients who received radiotherapy).

Fifty-five patients (90.2\%) received chemotherapy. Of these, 52 (94.5\%) had both neoadjuvant and post-adjuvant chemotherapy and 3 (5.5\%) post-adjuvant chemotherapy only. Palliative radiotherapy was administered to $2 / 61$ patients (3.3\%) who were not fit for surgery. Two patients developed local recurrence, of whom one was treated with amputation and the other with further excision.

At the time of the study, 59 patients $(96.7 \%)$ were traceable or known to have died. The two untraceable patients were from other countries and had been referred home for further treatment after limb salvage. Of the patients who were alive $(25 / 59,42.4 \%), 10 / 13$ (76.9\%) had been treated with limb salvage and 14/33 (42.4\%) with amputation, and the rest $(1 / 4,25.0 \%)$ had refused surgery. Of the $34 / 59$ patients who were dead, $1 / 11(9.1 \%)$ had been treated with limb salvage and 19/33 (57.6\%) with amputation, and $3 / 4$ had refused surgery (Table 2$)$. Of the $13 / 61$ patients $(21.3 \%$, including the two untraceable patients) who defaulted from follow-up or had refused treatment, 3/13 (23.1\%) had been treated with limb salvage and 9/33 with amputation (27.3\%).

Our 1-year survival rate was $62.7 \%$ (95\% confidence interval (CI) 49.1 - 73.9) and our 5-year survival rate $38.1 \%$ (95\% CI 24.6 51.4) (Fig. 1). Fig. 2 shows that there was a statistically significant

Table 2. Survival v. treatment options

\begin{tabular}{llllll}
\hline & Limb salvage & Amputation & Palliative & Refused treatment & Total \\
\hline Alive & 10 & 14 & 0 & 1 & 25 \\
Dead & 1 & 19 & 11 & 3 & 34 \\
Unknown & 2 & 0 & 0 & 0 & 2 \\
Total & 13 & 33 & 11 & 4 & 61
\end{tabular}




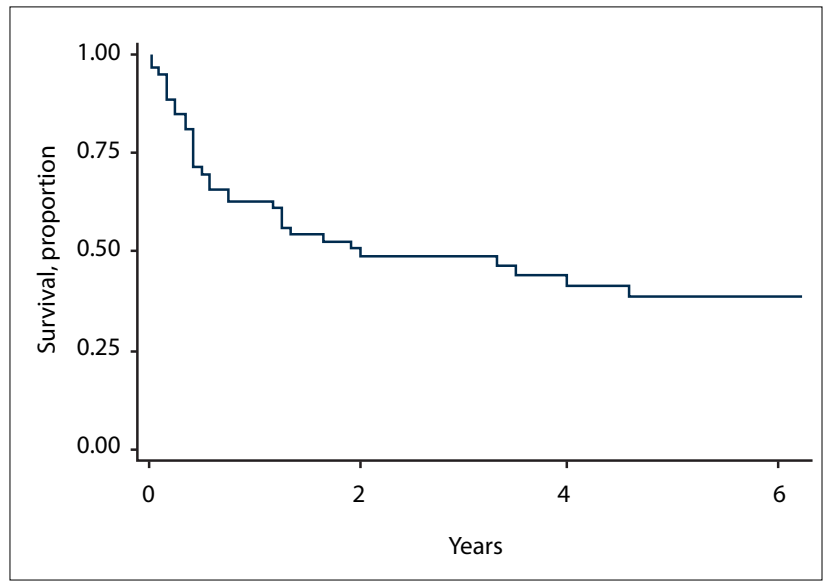

Fig. 1. Overall Kaplan-Meier survival estimate in years.

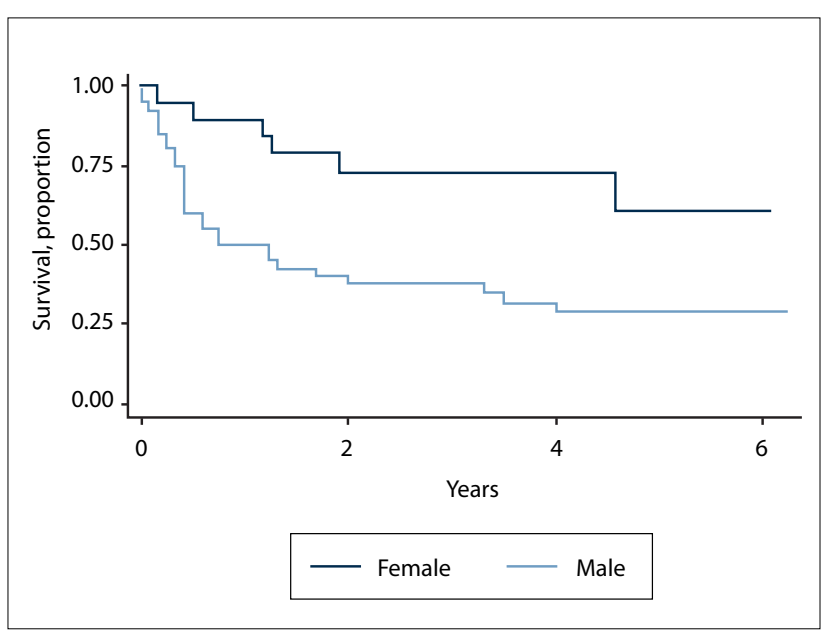

Fig. 2. Overall Kaplan-Meier survival estimates by sex.

difference between the survival curves for the two sexes $(p=0.009)$ as calculated by the log-rank test, with males being worse off. However, survival according to time (in months) before arrival at CMJAH did not differ significantly ( $p=0.587$ ) (Fig. 3 ).

All the patients who were staged as Enneking $2 \mathrm{~A}$ were alive at 5 years (range 5.08 - 6) without recurrences. Patients with Enneking stage $2 \mathrm{~B}$ disease had 1-year and 5-year survival rates of $69.2 \%$ (95\% CI 52.2 - 81.2) and 47.9\% (95\% CI 30.4 - 63.4), respectively. For patients with Enneking stage 3 disease, the 1- and 5-year survival rates were $37.5 \%$ (95\% CI 15.4 - 59.7) and 3.75\% (95\% CI 0.06 - 23.4), respectively (Fig. 4). This difference is statistically significant $(p=0.0008)$.

Only one patient who refused treatment was alive, 24 months after discharging herself from the hospital. She had presented with a 9-month history of a painful swollen knee and was subsequently diagnosed with conventional osteosarcoma of the proximal tibia, Enneking stage 2B.

Only one of the three patients who initially presented with a pathological fracture was alive, without recurrence at 54 months. She was a 12-year-old girl who had a pathological fracture of the left proximal tibia and was treated with above-knee amputation (AKA).

\section{Discussion}

Our 1-year and 5-year overall survival rates of $62.7 \%$ and $38.1 \%$ are better than those in previous published studies from developing countries. $^{[15-17]}$ Muthuphei and Maribas ${ }^{[15]}$ SA study in 2000 had 1 - and 5-year survival rates of $25.8 \%$ and $7.5 \%$, while Noor et al. ${ }^{[17]}$

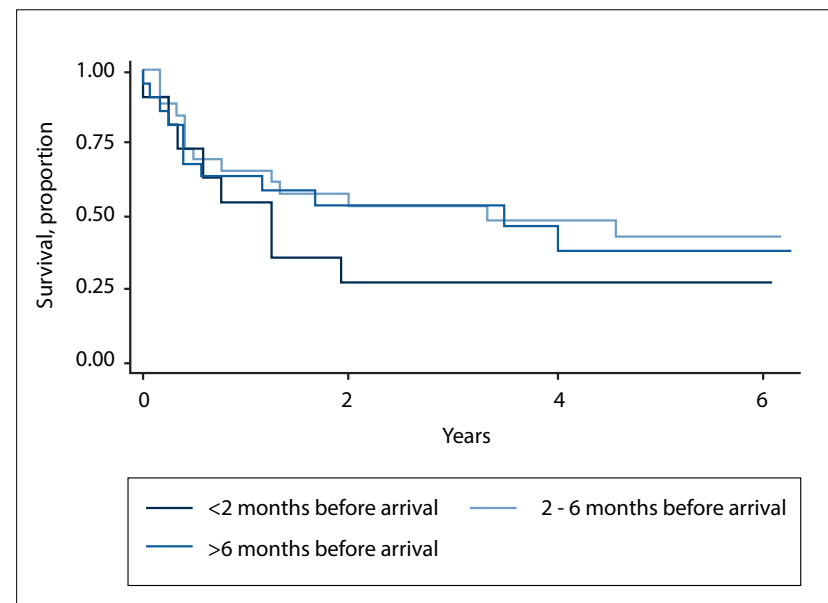

Fig. 3. Overall Kaplan-Meier survival estimates by time that elapsed before patients presented at Charlotte Maxeke Johannesburg Academic Hospital.

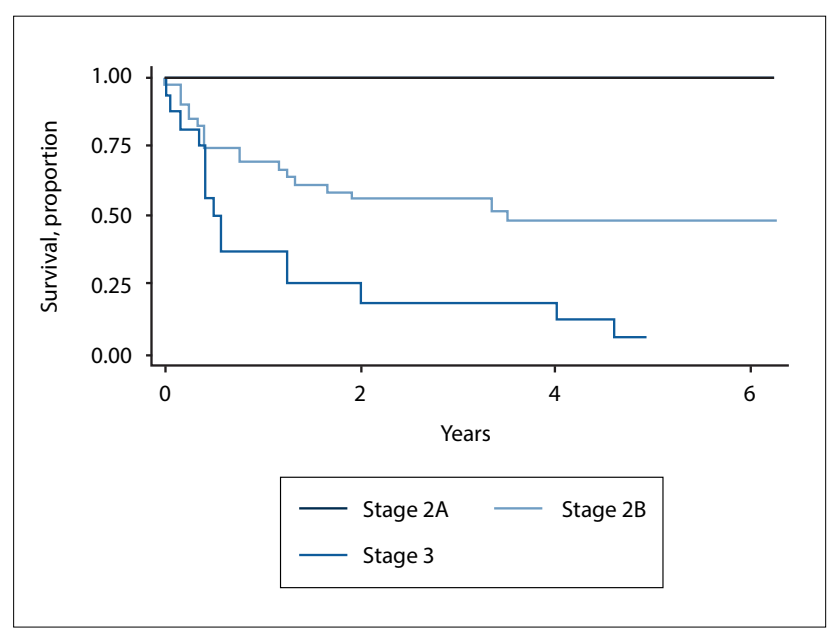

Fig. 4. Overall Kaplan-Meier survival estimates by Enneking stage.

reported 1- and 5-year survival of $53.6 \%$ and $8 \%$ in Cambodia. ${ }^{[15,17]}$ Our 5-year survival figure is worse than the corresponding figures for developed countries, which average $>50 \%{ }^{[18-20]}$ Several factors may account for our relatively poor outcomes. Our patients presented on average 4.5 months after the onset of symptoms, with Enneking stages $2 \mathrm{~B}$ and 3 accounting for a combined $93.4 \%$ compared with $6.6 \%$ with stage $2 \mathrm{~A}$. The delays may be attributable to poor accessibility of health facilities, especially for individuals living in rural areas. The higher the Enneking stage on presentation the poorer the prognosis, as has been reported in the literature ${ }^{[12]}$ and shown by our results. Although only $4.9 \%$ of patients admitted to first attending a traditional healer, most patients would have consulted them, as evidenced by the presence of 'traditional' scarifications. Almost a third of the patients came from outside our catchment area, which could have led to delays in referral. Limited access to MRI in our hospital, leading to a further average delay of 3 weeks, exacerbated the situation. Biopsies were done approximately a week after the MRI and other investigations were completed, resulting in an overall mean time to diagnosis of 23.5 weeks. In contrast, the median time to diagnosis in developed countries has been reported as 9 weeks. ${ }^{[21]}$ Patients from rural areas depend on doctors in district hospitals having a high index of suspicion of 'painful limbs' and doing initial investigations (X-rays) of the affected limb. Improving awareness through education of the community at large, and district doctors in 
particular, could increase early referral to specialised tumour units and hence the cure rate.

Amputees had poorer survival rates than patients who underwent limb salvage, because they had more advanced disease. Amputation was offered as 'palliation surgery' to relieve malaise and for pain control. More amputees than patients who had undergone limb salvage defaulted from follow-up at outpatient clinics (orthopaedic and oncology), despite multidisciplinary emphasis in hospital on surveillance for recurrence, which could explain their poor outcome. Preservation of the limb with limb salvage procedures, and hence a better self-image, could be a factor explaining the good attendance by these patients.

The majority of our patients (95.1\%) presented with a painful swollen limb and the rest with pathological fractures. Histological conventional osteosarcoma accounted for $93.4 \%$ of cases, while the rest (periosteal, pariosteal and telangiectatic) accounted for $6.6 \%$, as shown in Table 1. The incidence of pathological fractures is reported to be $5-10 \%$ in the literature ${ }^{[22-24]}$ Historically, pathological fractures were treated by amputation because of damage to the microcirculation that would result in contamination of adjacent soft tissue and metastasis. ${ }^{[21]}$ Several authors have since shown that there is no difference in terms of survival between patients who have amputation and those who have limb salvage. ${ }^{[22,24,25]}$ Three patients in our study presented with pathological fractures. Two of these patients, who had pathological fractures of the distal femur, had metastases and died in hospital from advanced disease. One patient, who was unfit for surgery, died few days after admission and the other patient, who underwent AKA, 2 months after admission. The only patient with a pathological fracture who survived was a 12 -year-old girl with high-grade osteosarcoma of the left distal tibia. She received neoadjuvant and post-adjuvant chemotherapy and was offered AKA because the tumour encapsulated the neurovascular structures. She was alive and free of disease free 54 months after AKA. Most of our patients presented with advanced disease precluding limb salvage.

Just over a quarter of our patients presented with metastases on admission. Our 5-year survival of 3.75\% for this cohort of patients is very low compared with the mid-20s - 30\% in developed countries, which is attributable to late presentation. ${ }^{[12]}$

One of the four patients who refused chemotherapy and surgery was traceable and alive 24 months after discharging herself. She was a 20-year-old woman with high-grade osteosarcoma of the left proximal tibia, Enneking stage $2 \mathrm{~B}$. She was being looked after by relatives at home.

\section{Study limitations}

Although $96.7 \%$ of our patients were traceable (or known to have died) at the time of the study, $\sim 20 \%$ of them had defaulted from follow-up. This is a major limiting factor in our study. This high default rate could be due to difficulty in accessing our facility, and also to the fact that $\sim 30 \%$ of our patients came from other provinces and countries. The majority of those who had defaulted cited lack of funds as the reason for not attending. Attempted telephonic contact of the two patients from other countries was unsuccessful as the numbers were not in use. Another limiting factor is the retrospective nature of the study, despite the fact that the data were collected prospectively using a comprehensive tumour form.

\section{Conclusion}

Treatment of osteosarcoma is challenging, demanding and timeconsuming, and requires meticulous record keeping. Limited resources hamper a multidisciplinary approach in developing countries. In our setting, both patients and their families need extensive education and counselling to improve access to modern treatments.

Despite better overall 1-year and 5-year survival rates than in other developing countries, our 5-year overall survival rates are suboptimal compared with developed countries, mainly because of delayed referral and logistical constraints to rapid work-up. We strongly recommend improved professional and public awareness of the importance of rapid referral, which is vital for the survival of these patients.

Acknowledgements. We thank Estelle Viljoen for keeping the database and fellow registrars for completing the tumour forms.

Author contributions. LL, ZAL, RDK and ML participated in the planning and design of the study and in interpretation of the results. LL wrote the first draft, and RK and ML edited it and supervised the project. All authors revised, edited and approved the final draft.

Funding. None.

Conflicts of interest. None.

1. PosthumaDeBoer J, Witlox MA, Kaspers GJ, van Royen BJ. Molecular alterations as target for therapy in metastatic osteosarcoma: A review of literature. Clin Exp Metastasis 2011;28(5):493-503. https://doi. in metastatic osteosarcoma: A review

2. Mirabello L, Troisi RJ, Savage SA. Osteosarcoma incidence and survival rates from 1973 to 2004. Cancer 2009;115(7):1531-1543. https://doi.org/10.1002/cncr.24121

2009;115(7):1531-1543. https://doi.org/10.1002/cncr.24121 3avage SA, Mirabello L. Using epidemiology and genomics to understan

Sarcoma 2011(2011), Article ID 548151. https://doi.org/10.1155/2011/548151

4. Dean BJ, Whitwell D. Epidemiology of bone and soft-tissue sarcomas. Orthop Trauma 2009;23(4):223230. https://doi.org/10.1016/j.mporth.2009.05.006

5. Geel J. Osteosarcoma: Current and future management in South Africa. S Afr Paediatr Rev 2009;6(3):32-39. https://www.researchgate.net/publication/239799371_Osteosarcoma_Current_and Future_Management_in_South_Africa (accessed 7 August 2017).

6. Duong LM, Richardson LC. Descriptive epidemiology of malignant primary osteosarcoma using population-based registries, United States, 1999 - 2008. J Registry Manag 2013;40(2):59-64.

Mirabello L, Troisi RJ, Savage SA. International osteosarcoma incidence patterns in hildren and Mirabello $\mathrm{L}$, Troisi $\mathrm{R}$, Savage SA. International osteosarcoma incidence patterns in children and adolescents, 24320

8. Hansen MF. Genetic and molecular aspects of osteosarcoma. J Musculoskelet Neuronal Interact 2002;2(6):554-560

9. Martin JW, Squire JA, Zielenska M. The genetics of osteosarcoma. Sarcoma 2012(2012), Article Martin JW, Squire JA, Zielenska M. The gen

ID 627254. https://doi.org/10.1155/2012/627254
0. Coventry MB, Dahlin DC. Osteogenic sarcoma. J Bone Joint Surg Am 1957;39(4):741-758.

11. Rosen G. Preoperative (neoadjuvant) chemotherapy for osteogenic sarcoma: A ten year experience. Orthopedics 1985;8(5):659-964. https://doi.org/10.3928/0147-7447-19850501-19

12. Allison DC, Carney SC, Ahlmann ER, et al. A meta-analysis of osteosarcoma outcomes in the modern medical era. Sarcoma 2012(2012), Article ID 704872. https://doi.org/10.1155/2012/704872

13. Li X, Ashana AO, Moretti VM, Lackman RD. The relation of tumour necrosis and survival in patients with osteosarcoma. Int Orthop 2011;35(12):1847-1853. https://doi.org/10.1007/s00264-011-1209-7

14. Li X, Moretti VM, Ashana AO, Lackman RD. Impact of close surgical margin on local recurrence and survival in osteosarcoma. Int Orthop 2012;36(1):131 -137. https://doi.org/10.1007/s00264-011-1230-x

15. Muthuphei MN, Mariba MT. Osteosarcoma in Ga-Rankuwa Hospital: A 10-year experience in an African population. Cent Afr J Med 2000;46(2):41-43.

16. Shipley JA, Beukes CA. Outcomes of osteosarcoma in a tertiary hospital. SA Orthop J 2012;11(1):18-22. http://www.scielo.org.za/pdf/saoj/v1 ln1/03.pdf (accessed 7 August 2017).

7. Noor S, Pormóðsson HS, Zervas CT, Ly T, Gollogly J. Limb versus life - the outcomes of osteosarcoma in Cambodia. Int Orthop 2014;38(3):579-585. https://doi.org/10.1007/s00264-013-2173-1

18. Henshaw RM, Priebat DA, Perry DJ, Shmookler BM, Malawer MM. Survival after induction chemotherapy and surgical resection for high-grade soft tissue sarcoma. Is radiation necessary? Ann Surg Oncol 2001;8(6):484-495. https://doi.org/10.1007/s00264-013-2173-1

19. Goorin AM, Perez-Atayde A, Gebhardt M, et al. Weekly high-dose methotrexate and doxorubicin for osteosarcoma: The Dana-Farber Cancer Institute/the Children's Hospital--study III. J Clin Oncol 1987;5(8):1178-1184. https://doi.org/10.1200/JCO.1987.5.8.1178

20. Bacci G, Ferrari S, Bertoni F, et al. Long-term outcome for patients with nonmetastatic osteosarcoma of the extremity treated at the Istituto Ortopedico Rizzoli according to the Istituto Ortopedico Rizzoli/ Osteosarcoma-2 protocol: An updated report. J Clin Oncol 2000;18(24):4016-4027. https://doi. org/10.1200/JCO.2000.18.24.4016

21. Brasme JF, Morfouace M, Grill J, et al. Delays in diagnosis of paediatric cancers: A systematic review and comparison with expert testimony in lawsuits. Lancet Oncol 2012;13(10):e445-e459. https://doi. arg comparton whe expert testing

22. Abudu A, Sferopoulos NK, Tillman RM, Carter SR, Grimer RJ. The surgical treatment and outcome of Abudu A, Sferopoulos NK, Tillman RM, Carter SR, Grimer RJ. The surgical treatment an
pathological fractures in localised osteosarcoma. J Bone Joint Surg Br 1996;78(5):694-698.

pathological fractures in localised osteosarcoma.J Bone Joint Surg Br 1996;78(5):694-698.
23. Jaffe N, Spears R, Eftekhari F, et al. Pathologic fracture in osteosarcoma: Impact of chemotherapy on primary tumor and survival. Cancer 1987;59(4):701-709. https://doi.org/10.1002/10970142(19870215)59:4<701::AID-CNCR2820590407>3.0.CO;2-V

24. Bacci G, Ferrari S, Longhi A, et al. Nonmetastatic osteosarcoma of the extremity with pathologic fracture at presentation: Local and systemic control by amputation or limb salvage after preoperative chemotherapy. Acta Orthop Scand 2003;74(4):449-454. https://doi.org/10.1080/00016470310017776

25. Scully SP, Ghert MA, Zurakowski D, Thompson RC, Gebhardt MC. Pathologic fracture in osteosarcoma. I Bone Joint Surg Am 2002;84(1):49-57.

Accepted 7 April 2017 